\title{
DOES QUANTITATIVE LEFT VENTRICULAR REGIONAL WALL MOTION CHANGE AFTER FIBROUS TISSUE RESECTION IN ENDOMYOCARDIAL FIBROSIS?
}

\author{
Vera Maria Cury Salemi, ${ }^{\mathrm{I}}$ Fabio Fernandes, ${ }^{\mathrm{I}}$ Raquel Sirvente, ${ }^{\mathrm{I}}$ Luciano \\ Nastari, ${ }^{\mathrm{I}}$ Leonardo Vieira Rosa, ${ }^{\mathrm{I}}$ Cristiano A. Ferreira, ${ }^{\mathrm{I}} \mathrm{José} \mathrm{Luiz} \mathrm{Barros} \mathrm{Pena,}$ \\ I Michael H. Picard, "I Charles Mady ${ }^{\mathrm{I}}$
}

doi: $10.1590 / \mathrm{S1807-59322009000100004}$

Salemi VMC, Fernandes F, Sirvente R, Nastari L, Rosa LV, Ferreira CA, Pena JLB, Picard MH, Mady C. Does quantitative left ventricular regional wall motion change after fibrous tissue resection in endomyocardial fibrosis? Clinics. 2009;64(1): $17-22$

OBJECTIVES: We compared left ventricular regional wall motion, the global left ventricular ejection fraction, and the New York Heart Association functional class pre- and postoperatively.

INTRODUCTION: Endomyocardial fibrosis is characterized by fibrous tissue deposition in the endomyocardium of the apex and/or inflow tract of one or both ventricles. Although left ventricular global systolic function is preserved, patients exhibit wall motion abnormalities in the apical and inferoapical regions. Fibrous tissue resection in New York Heart Association FC III and IV endomyocardial fibrosis patients has been shown to decrease morbidity and mortality.

METHODS: We prospectively studied 30 patients ( 20 female, $30 \pm 10$ years) before and $5 \pm 8$ months after surgery. The left ventricular ejection fraction was determined using the area-length method. Regional left ventricular motion was measured by the centerline method. Five left ventricular segments were analyzed pre- and postoperatively. Abnormality was expressed in units of standard deviation from the mean motion in a normal reference population.

RESULTS: Left ventricular wall motion in the five regions did not differ between pre- and postoperative measurements. Additionally, the left ventricular ejection fraction did not change after surgery $(0.45 \pm 0.13 \%$ x $0.43 \pm 0.12 \%$ pre- and postoperatively, respectively). The New York Heart Association functional class improved to class I in $40 \%$ and class II in $43 \%$ of patients postoperatively $(\mathrm{p}<0.05)$.

CONCLUSIONS: Although endomyocardial fibrosis patients have improved clinical symptoms after surgery, the global left ventricular ejection fraction and regional wall motion in these patients do not change. This finding suggests that other explanations, such as improvements in diastolic function, may be operational.

KEYWORDS: Endomyocardial fibrosis; Wall motion; Restrictive cardiomyopathy; Ejection fraction; Surgery.

\section{INTRODUÇÃO}

The clinical and pathological features of endomyocardial fibrosis (EMF) were first described by Jack N. P. Davies in

${ }^{\text {I }}$ Cardiomyopathy Unit, Heart Institute (InCor), Hospital das Clínicas, Faculdade de Medicina, Universidade de São Paulo/ SP, Brazil.

II Cardiology Division and Cardiac Ultrasound Laboratory, Massachusetts General Hospital and Harvard Medical School - Boston/MA, USA.

Email: verasalemi@uol.com.br

Tel.: 55.11 3556.9832

Received for publication on July 03, 2008

Accepted for publication on September 15, 2008
1948. ${ }^{1}$ EMF is a restrictive cardiomyopathy characterized by fibrous tissue (FT) deposition in the endomyocardium of the apex and/or inflow tract of one or both ventricles. ${ }^{2}$ The involvement of the subvalvar apparatus by FT may cause atrioventricular regurgitation. Ventricular volumes are usually normal or decreased by FT deposition, whereas atrial volumes are enlarged and reflect diastolic dysfunction. ${ }^{3}$ Clinical manifestation occurs as right or left cardiac failure, depending on the degree of bilateral ventricle involvement. ${ }^{4}$ Diagnosis may be performed noninvasively by echocardiography ${ }^{3}$ or magnetic resonance imaging ${ }^{5}$ via recognition of apical 
obliteration, atrioventricular valve regurgitation, and diastolic dysfunction or, more rarely, by invasive contrast ventriculography. ${ }^{6}$ Although the global LV ejection fraction (EF) is preserved in these patients, a previous study by our group showed that patients exhibit wall motion abnormalities in the apical and inferoapical regions that are unrelated to the amount of FT deposition. ${ }^{6}$ Treatment for patients in the New York Heart Association (NYHA) functional classes (FC) III and IV who are unresponsive to clinical treatment involves surgery. ${ }^{7}$ The surgical treatment consists of FT resection and repair or replacement of atrioventricular valves. This has been shown to decrease both morbidity and mortality. ${ }^{8}$ It has been previously reported that surgical treatment improves contractile myocardial function, ${ }^{9}$ although quantitative assessment of both global and regional myocardial function before and after FT resection has not been performed. Therefore, the objective of our study was to quantitatively compare LV regional wall motion, the global LVEF, and the NYHA FC pre- and postoperatively in EMF patients.

\section{METHODS}

We prospectively studied 30 patients (20 female, $30 \pm 10$ years) with LV EMF before and $5 \pm 8$ months after surgery. Seventeen patients (57\%) had moderate to severe RV EMF associated with LV EMF. Thirteen (43\%) patients were classified in NYHA FC IV and 17 (57\%) patients in NYHA FC III. The diagnosis of EMF was based on clinical presentation as well as electrocardiographic and echocardiographic data. The typical echocardiographic examination showed apical obliteration, atrial dilation, and variable degrees of atrioventricular valve regurgitation. ${ }^{3}$ Fourteen (47\%) patients underwent LV FT resection, and 16 (53\%) underwent biventricular FT resection. Additionally, three patients $(10 \%)$ underwent mitral valve replacement, one $(3 \%)$ tricuspid replacement, five $(17 \%)$ mitral valve repair, seven (23\%) tricuspid valve repair, and 12 (40\%) mitral and tricuspid repairs. The angiographic images of the LV were performed using a pigtail catheter in the anterior right oblique projection at $30^{\circ}$, and these images confirmed the EMF diagnosis. ${ }^{10}$ The LVEF was determined by the area-length method, ${ }^{11,12}$ and the regional $\mathrm{LV}$ wall motion was quantified using the centerline method. ${ }^{13,14}$ Contrast images representing the LV in maximum systole and diastole were selected, and their contours were traced onto paper by hand for later calculations. The images were analyzed by two independent observers who were blinded to identity of the patients, as previously described. ${ }^{6}$ Using a specific program, ${ }^{15}$ the ventricular images were recorded in a microcomputer with the aid of a digitalizing table (CURTA/model IS/ONE). The centerline was constructed in the computer between the end-diastolic and end-systolic contours. Motion was measured in 100 equidistant chords perpendicular to the centerline. The length of each chord indicated the motion of the corresponding point in the LV contour. The chords were normalized to cardiac size by dividing each length by the final diastolic margin, and a unit without dimensions was obtained. Abnormality was expressed as units of standard deviation from the mean motion of the normal reference population comprised of 103 individuals (52 men, $52 \pm 10$ years) with normal LV according to clinical, electrocardiographic, and angiographic data. Positive values indicated hyperkinesia, and negative values indicated hypokinesia. Chord measurement was initiated at the level of the aortic valve, and the chords were successively numbered in a clockwise direction from the aortic valvular plane to the mitral plane. ${ }^{16}$ The regions from 1 to 10 demarcated by the chords were excluded because their analysis revealed great variation in length. Chords 81 to 100 were also excluded since they represented the mitral valve. ${ }^{16}$ The LV was divided into five regions: anterobasal $(\mathrm{AB}$, chords 10 to 23), anterolateral (AL, chords 24 to 37), apical (A, chords 38 to 52), inferoapical (IA, chords 53 to 66), and inferobasal (IB, chords 67 to 80) (Figure 1).

Our Institutional Review Board approved this study, and informed consent was obtained from all patients.

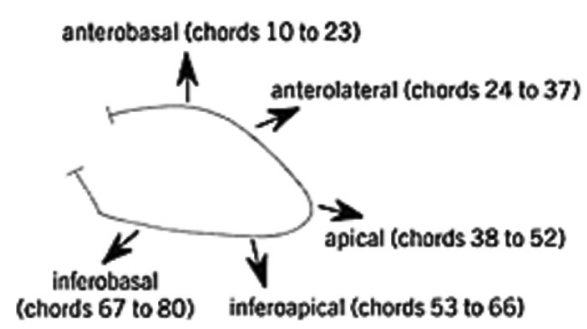

Figure 1 - The left ventricle was divided into five regions: anterobasal: chords 10 to 23 , anterolateral: chords 24 to 37 , apical: chords 38 to 52, inferoapical: chords 53 to 66 , and inferobasal: chords 67 to 80 . Chords 1 to 10 were excluded due to their high variability in length, and chords 81 to 100 were excluded since they represent the mitral valve

\section{Statistical analyses}

Paired t-tests were used to compare regional LV wall motion and the LV ejection fraction before and after surgery. The Mann-Whitney test was used to compare the NHYA functional class pre- and postoperatively. A probability (P) value less than 0.05 was considered statistically significant. Statistical analyses were performed using SPSS v 15 (SPSS Inc. Chicago, IL, USA).

\section{RESULTS}

Quantitatively, LV wall motion in the five regions did not 
differ between the pre- and postoperative time points $(\mathrm{AB}$ : pre $-0.78 \pm 0.25$ x post $-0.96 \pm 0.24, p=0.559$; AL: pre -0.90 $\pm 0.33 \mathrm{x}$ post $-1.26 \pm 0.26, \mathrm{p}=0.275 ; \mathrm{A}$ : pre $-1.84 \pm 0.31 \mathrm{x}$ post $-1.94 \pm 0.34, \mathrm{p}=0.732$; IA: pre $-1.69 \pm 0.29 \mathrm{x}$ post -2.23 $\pm 0.39, \mathrm{p}=0.158$, IB: pre $-0.92 \pm 0.21 \mathrm{x}$ post $-1.24 \pm 0.30, \mathrm{p}=$ 0.347; Figure 2). Similarly, the LVEF did not change after surgery $(0.45 \pm 0.13 \% \times 0.43 \pm 0.12$ pre- and postoperatively, respectively). However, the NYHA FC was classified as III in 17 patients (57\%) and IV in 13 patients (43\%), and it improved to I in $40 \%$ and II in $43 \%$ of the patients postoperatively ( $<<0.05$, Figure 3 ). Seventeen percent of the postoperative patients remained in NYHA FC III and IV.

\section{DISCUSSION}

This report demonstrates that LVEF and quantitative regional wall motion do not change even though EMF patients show improvements in their clinical symptoms after surgery. Regional wall motion has been shown to be present in EMF patients in A and IA regions, ${ }^{6}$ and we demonstrate herein that the wall motion abnormalities do not change after FT resection. In histopathological specimens obtained from surgical resection, the FT extends irregularly into the underlying myocardium ${ }^{17}$ and separates groups of myocardial fibers. Additionally, FT is composed of a superficial compact

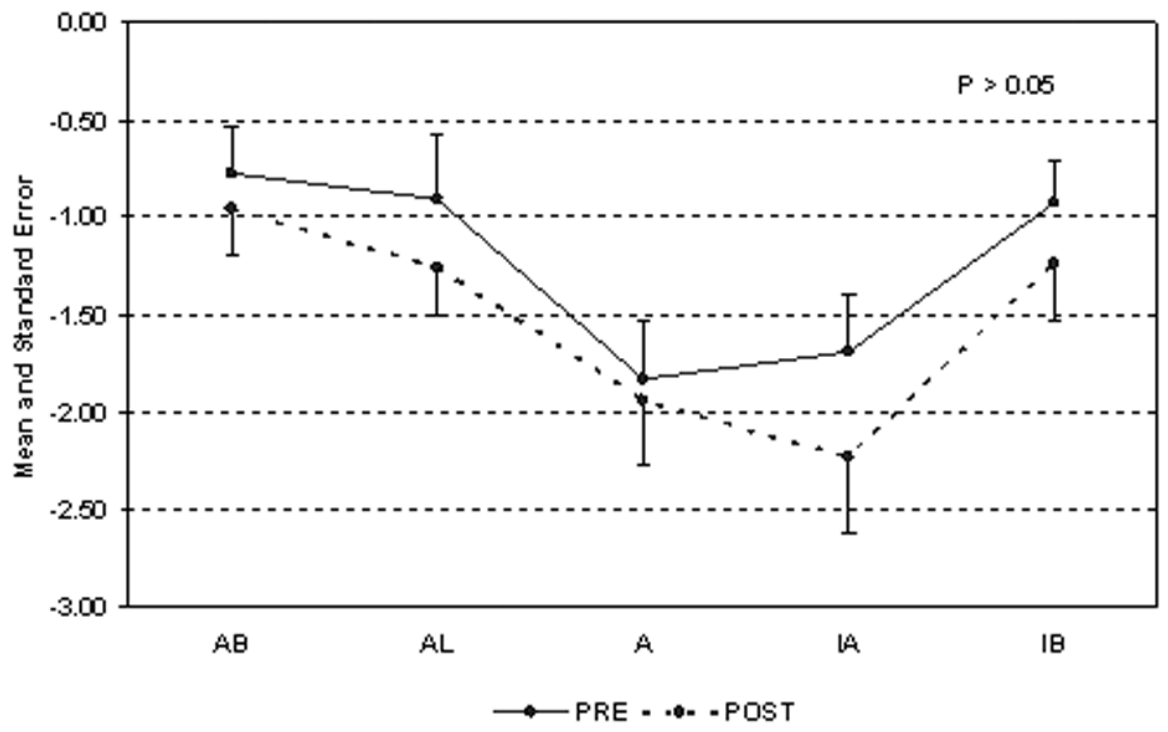

Figure 2 - Quantitatively, LV wall motion in the five regions did not differ pre- and postoperatively in EMF patients; $\mathrm{AB}$ - anterobasal; $\mathrm{AL}$ - anterolateral; A - apical; IA - inferoapical; IB - inferobasal

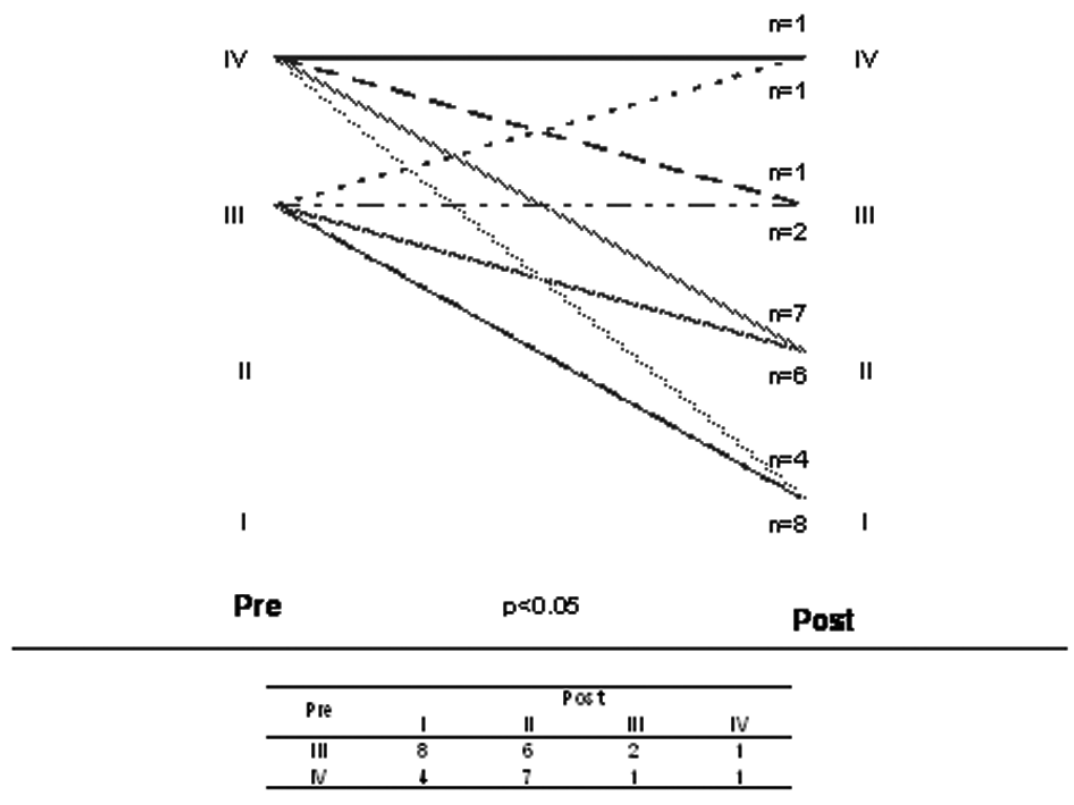

Figure 3 - The NYHA functional class improved to I in $40 \%$ and II in $43 \%$ of the patients postoperatively 
layer of collagen and a deeper layer of loose FT, and the myocardial fibers commonly show degenerative changes. The apical and inferoapical wall motion abnormalities are maintained as the FT is resected, likely by FT that penetrates into the myocardium and/or myocytes that have degenerated. Our findings are reinforced by the mildly depressed LVEF that does not change after resection. In contrast to previous studies affirming that surgery improves contractile function by releasing the myocardium, ${ }^{9,18}$ no changes in global or regional functions were observed in our study. In contrast to previous work, we used a quantitative technique to compare regional wall motion in EMF patients before and after surgical resection.

However, the high rates of symptom improvement require an explanation. It is likely that FT resection causes an increase in LV volumes, which may improve ventricular filling, produce a less steep pressure-volume relationship, and improve diastolic function. We have previously shown that EMF patients exhibit delayed myocardial relaxation, increased ventricular stiffness, and enlarged left atrium volumes. ${ }^{3}$ Potential changes in diastolic function after FT resection were not assessed, although the present study suggests that further evaluation of such changes would be valuable.

In our study, surgery resulted in an impressive response, as measured by the NYHA FC. We have previously shown that the NYHA FC is strongly related to maximum oxygen consumption in EMF patients ${ }^{19,20}$ and is a major factor in the decision for surgical indication. ${ }^{10}$ Early surgery is the only intervention that may reduce morbidity and improve survival in patients presenting NYHA FC III and IV. ${ }^{10}$ Medically treated symptomatic patients have a mortality rate of $50 \%$ over 2 years, ${ }^{21}$ whereas the operative mortality rate is about $15 \%$ to $20 \%$. Furthermore, the actuarial probability of survival at 17 years post-treatment is $55 \%$ when operative mortality is considered. ${ }^{22}$

The improvements in morbidity and mortality observed with surgical treatment are only achieved in symptomatic patients. Clinical treatment of EMF patients as well as patients with other restrictive cardiomyopathies is complex, because hypovolemia or hypervolemia can lead to hypotension or pulmonary congestion, respectively. ${ }^{23}$ Additionally, medical treatments must be used with caution because vasodilators may cause hypotension and medications that decrease heart rate in an attempt to improve ventricular filling have not proved beneficial. ${ }^{23}$

EMF is a "neglected" disease in cardiology because it almost exclusively affects the poorest people in developed countries. ${ }^{24}$ EMF is still prevalent in tropical areas of South America, Africa, and Asia, and over 12 million people are currently estimated to have EMF. ${ }^{25}$ Endomyocardial fibrosis is found in $9 \%$ of the general population in affected regions of Mozambique, ${ }^{9}$ accounting for $15 \%$ to $25 \%$ of all cardiac deaths in equatorial Africa. Unfortunately, EMF most severely affects those regions least equipped for cardiovascular surgery. ${ }^{25}$

The cause and pathogenesis of EMF remain unknown. The unifying explanation considers EMF and Loeffler's syndrome to be extremes of the same disease due to the similarity between the heart lesions in both disorders. ${ }^{26}$ Several variables, including environmental (geography, nutritional deficiency, high cerium intake, and parasitic infectious) and host (genetic predisposition, ethnicity, hypereosinophilia, autoimmunity, and inflammatory processes) factors, are involved in the pathogenesis of EMF. ${ }^{25-27}$ Of note, eosinophils remain a major risk factor for EMF in Uganda and Nigeria, but their role in the pathogenesis of this disease is still not understood. ${ }^{28}$

The age and sex predominance of EMF vary depending on the region considered. As observed in our study, EMF mainly affects young female adults in Brazil. Male predominance occurs in Kerala and Nigeria, whereas the disease strikes primarily female patients in Uganda. ${ }^{25}$ The incidence in Africa peaks in people between the ages of 11 and 15 years. ${ }^{9}$ In Uganda, bimodal peaks occur at age 10 and age $30 .{ }^{26}$ In contrast, heart failure in developed countries is a disease of the elderly. ${ }^{29}$

Ventricular involvement depends on the geographic area. In our hospital, $52.4 \%$ of the patients had biventricular involvement, $27.9 \% \mathrm{RV}$ involvement, and $30.7 \% \mathrm{LV}$ involvement. ${ }^{30} \mathrm{RV}$ and biventricular involvement are more common in Africa. ${ }^{9}, 31$

\section{CONCLUSION}

Even though EMF patients show improvements in clinical symptoms after surgery, the LVEF and quantitative regional wall motion do not change. These results suggest that the improvements in symptoms are due to improvements in diastolic function.

\section{ACKNOWLEDGMENTS}

We thank Nivaldo Bertozzo Jr. for the acquisition of left ventricular image data and Julia Fukushima for performing the statistical analysis. 


\section{REFERENCE}

1. Davies JPN. Endomyocardial fibrosis in Uganda. East Afr Med J. 1948;25:10-16.

2. Ball JD, Williams AW, Davies JN. Endomyocardial fibrosis. Lancet. 1954;266:1049-54

3. Salemi VM, Picard MH, Mady C. Assessment of diastolic function in endomyocardial fibrosis: value of flow propagation velocity. Artif Organs. 2004;28:343-6.

4. Mady C, Barretto AC, Oliveira SA, Stolf N, Bellotti G, Pileggi F. Evolution of the endocardial fibrotic process in endomyocardial fibrosis. Am J Cardiol. 1991;68:402-3.

5. Salemi VM, Rochitte CE, Barbosa MM, Mady C. Clinical and echocardiographic dissociation in a patient with right ventricular endomyocardial fibrosis. Heart. 2005;91:1399.

6. Mady C, Salemi VM, Ianni BM, Arteaga E, Fernandes F, Ramires FJ. Quantitative assessment of left ventricular regional wall motion in endomyocardial fibrosis. Arq Bras Cardiol. 2005;84:241-4.

7. Mady C, Pereira Barretto AC, de Oliveira SA, Stolf NA, Bellotti G, Jatene AD, et al. Effectiveness of operative and nonoperative therapy in endomyocardial fibrosis. Am J Cardiol. 1989;63:1281-2.

8. de Oliveira SA, Pereira Barreto AC, Mady C, Dallan LA, da Luz PL, Jatene AD, et al. Surgical treatment of endomyocardial fibrosis: a new approach. J Am Coll Cardiol. 1990;16:1246-51.

9. Mayosi BM. Contemporary trends in the epidemiology and management of cardiomyopathy and pericarditis in sub-Saharan Africa. Heart. 2007;93:1176-83.

10. Barretto AC, da Luz PL, de Oliveira SA, Stolf NA, Mady C, Bellotti G, et al. Determinants of survival in endomyocardial fibrosis. Circulation. 1989;80:I177-82.

11. Dodge HT, Sandler H, Ballew BH, Load JD. The use of biplane angiocardiography for the measurement of left ventricular volume in man. Am Heart J. 1960;60:762-76.

12. Dodge HT, Sandler H, Baxley WA, Hawley RR. Usefulness and limitations of radiographic methods for determining left ventricular volume. Am J Cardiol. 1966;18:10-24.

13. Sheehan FH, Dodge HT, Mathey D, Brown BG, Bolson EL, Mitten S. Application of the centerline method: Analysis of change in regional left ventricular wall motion in serial studies. Comput Cardiol. 1982;3:97100 .

14. Sheehan FH, Bolson EL, Dodge HT, Mathey DG, Schofer J, Woo HW. Advantages and applications of the centerline method for characterizing regional ventricular function. Circulation. 1986;74:293-305.
15. Toscano M, Calderolli DM, Moura L, Melo CP, Bellotti G, Pileggi F. A microcomputer-based system for the analysis of left ventricle function. Automedica. 1992;15:163-75.

16. Sheehan FH, Stewart DK, Dodge HT, Mitten S, Bolson EL, Brwon BG. Variability in the measurement of regional left ventricular wall motion from contrast angiograms. Circulation. 1983;68:550-9.

17. Iglezias SD, Benvenuti LA, Calabrese F, Salemi VM, Silva AM, Carturan E, et al. Endomyocardial fibrosis: pathological and molecular findings of surgically resected ventricular endomyocardium. Virchows Arch. 2008;453:233-41

18. Mocumbi AO, Sidi D, Vouhe P, Yacoub M. An innovative technique for the relief of right ventricular trabecular cavity obliteration in endomyocardial fibrosis. J Thorac Cardiovasc Surg. 2007;134:10702 .

19. Mady C, Barretto AC, Mesquita ET, Silva PR, Cardoso RH, Bellotti $\mathrm{G}$, et al. Maximal functional capacity in patients with endomyocardial fibrosis. Eur Heart J. 1993;14:240-2.

20. Salemi VM, Leite JJ, Picard MH, Oliveira LM, Reis SF, Pena JLB, et al. Echocardiographic predictors of functional capacity in endomyocardial fibrosis patients. Eur J Echocardiogr. 2008, in press.

21. D’Arbela PG, Mutazindwa T, Patel AK, Somers K. Survival after first presentation with endomyocardial fibrosis. Br Heart J. 1972;34:4037.

22. Moraes F, Lapa C, Hazin S, Tenorio E, Gomes C, Moraes CR. Surgery for endomyocardial fibrosis revisited. Eur J Cardiothorac Surg. 1999; $15: 309-12$

23. Desai A, Fang JC. Heart failure with preserved ejection fraction: hypertension, diabetes, obesity/sleep apnea, and hypertrophic and infiltrative cardiomyopathy. Heart Fail Clin. 2008;4:87-97.

24. Sliwa K, Damasceno A, Mayosi BM. Epidemiology and etiology of cardiomyopathy in Africa. Circulation. 2005;112:3577-83.

25. Mocumbi AO, Yacoub S, Yacoub MH. Neglected tropical cardiomyopathies: II. Endomyocardial fibrosis: myocardial disease. Heart. 2008;94:384-90.

26. Bukhman G, Ziegler J, Parry E. Endomyocardial fibrosis: still a mystery after 60 years. PLoS Neg1 Trop Dis. 2008;2:e97.

27. Shaper AG. The aetiology of endomyocardial fibrosis. In: Valiathan MS Somers K, Kartha CC, eds. Endomyocardial fibrosis, 1st ed. Oxford, England: Oxford University Press, 1993:113.

28. Rutakingirwa M, Ziegler JL, Newton R, Freers J. Poverty and eosinophilia are risk factors for endomyocardial fibrosis (EMF) in Uganda. Trop Med Int Health. 1999;4:229-35. 
29. Goldberg RJ, Ciampa J, Lessard D, Meyer TE, Spencer FA. Long-term survival after heart failure: a contemporary population-based perspective. Arch Intern Med. 2007;167:490-6.

30. Barretto AC, Mady C, Pileggi F. Endomyocardial fibrosis. Longitudinal follow-up of patients not treated with surgery. Arq Bras Cardiol. 1996;67:285-8.
31. Mocumbi AO, Ferreira MB, Sidi D, Yacoub MH. A population study of endomyocardial fibrosis in a rural area of Mozambique. N Engl J Med. 2008. 3;359:43-9. 